\title{
COMMENT
}

\section{Master manipulators: how herpesviruses alter immune responses to RSV}

\author{
Stephen J. Gurczynski ${ }^{1}$ and Bethany B. Moore (iD ${ }^{1,2}$
}

In this commentary on "A gammaherpesvirus licenses CD8 T cells to protect the host from pneumovirus-induced immunopathologies", the authors highlight the growing literature suggesting that herpesvirus infections shape subsequent immune responses to other pathogens, especially by broadening CD8+T-cell responses. These observations have implications for vaccine development against other important pathogens, such as Respiratory Syncytial Virus.

Mucosal Immunology (2020) 13:715-716; https://doi.org/10.1038/s41385-020-0313-7

\section{COMMENTARY}

From cell death and differentiation to immunity, herpesviruses have adapted to modulate almost every aspect of the host cellular systems in which they replicate. Due to their large genomes which encode for dozens of immune-modulatory proteins, herpesviruses are unique in their ability to manipulate immunity to both further their own replication and to alter host responses to other pathogens. Herpesviruses are also nearly ubiquitous in the human population, with some, such as Epstein Barr Virus (EBV) infecting upwards of $70-80 \%$ of the population by adulthood. Further, the lifelong nature of herpesvirus infections means that latent infections can alter immune responses long after primary acquisition. Thus, understanding how herpesviruses alter immune responses to secondary viral infections is critical to understanding disease outcomes, especially for viruses such as Respiratory Syncytial Virus (RSV), which can have disparate clinical manifestations ranging from asymptomatic all the way to life threating pulmonary infections. $^{1}$

Using a murine model of co-infection with gammaherpesvirus68 ( $ү \mathrm{HV}-68$ ) and murine pneumovirus (PVM) to simulate infection with both EBV and RSV in humans respectively, Dourcy et al. report that mice preinfected with $\mathrm{yHV}-68$ had an altered immune response to a subsequent PVM infection. Specifically, yHV-68 infected mice exhibited a decreased Th2 response, which in the case of PVM and RSV, represents the main immunologic mechanism of virus-induced pathology. Further, $\mathrm{yHV}-68$ infection preserved and even bolstered IFN $\gamma$-mediated immune responses, especially from memory CD8+ lymphocytes, which limited PVM replication. The balance of cytokines struck in this model, i.e., decreased Th2 response with a preserved IFNY response, is especially interesting given that RSV infection in children is associated with a lack of a protective IFN $/ / T h 1$ response and instead can result in an aberrant activation of IL-33 which leads to a robust IL-4/IL-13 (Th2) response and in some cases an IL-17 response that has been associated with the development of childhood asthma. ${ }^{2}$ Importantly, CD8 + responses are also thought to be ineffective and possibly pathogenic during RSV infection. ${ }^{3}$
Thus, the observation that herpesviruses drive the generation of a protective CD8 + centric response that effectively mediates RSV/ PVM clearance and does not cause overt pathology is especially intriguing.

Interestingly, even though this same group demonstrated that pulmonary $\mathrm{YHV}-68$ infection greatly alters both the monocytic and alveolar macrophage compartments, ${ }^{4}$ these cell types were not found to confer a protective advantage to PVM challenge. Together, these data suggest that herpesviruses can drive the maturation of memory CD8+ lymphocytes which confer a sterilizing mucosal immune response that can protect from subsequent infection with an unrelated virus. Further, even though these memory CD8+ cells are generated as a result of the initial herpesvirus infection, they show specificity for PVM epitopes indicating that herpesviruses can generate lymphocytes with a broad antigen repertoire which may have implications in RSV vaccine development. This result also highlights the potential benefit of herpesvirus infections in bolstering protective responses against other pathogens as well.

It was discovered nearly 15 years ago that latent gammaherpesvirus infection can alter antibacterial responses in mice. Barton et al. used a model of $\mathrm{yHV}-68$ preinfection and demonstrated that mice exhibited cross-protection against a lethal Yersinia pestis challenge suggesting that the protective effects of herpesvirus infection may not be solely limited to viral challenges. ${ }^{5}$ Interestingly, the protection in this model was also driven in large part by memory CD8+ lymphocytes secreting IFN $\gamma$. More recently, it was shown that gammaherpesvirus latency confers a protective response against lymphocytic choriomeningitis virus infection through a similar memory CD8+ lymphocyte driven mechanism. ${ }^{6}$

Further, this ability to modulate immune responses is not limited to gamma herpesviruses. Several groups are studying how to co-opt the immune-modulatory properties of other herpesviruses, specifically the beta herpesvirus cytomegalovirus, to generate enhanced vaccine responses. ${ }^{7}$ In a somewhat different take on the co-infection model, Simian immunodeficiency virus (SIV) proteins, specifically Gag and Env, have been cloned into a

\footnotetext{
${ }^{1}$ Division of Pulmonary and Critical Care Medicine, Department of Internal Medicine, University of Michigan, Ann Arbor, MI, USA and ${ }^{2}$ Department of Microbiology and Immunology, University of Michigan, Ann Arbor, MI, USA

Correspondence: Bethany B. Moore (bmoore@umich.edu)
}

Received: 1 May 2020 Revised: 13 May 2020 Accepted: 27 May 2020

Published online: 11 June 2020 
Rhesus cytomegalovirus (RhCMV) vector and used to immunize rhesus monkeys against SIV. This strategy elicits the generation of CD8 + T cells that break traditional epitope paradigms and can produce broadly neutralizing responses to epitopes of viral proteins that are not normally accessible. ${ }^{8}$ Interestingly, Dourcy et al. reported that at least a portion of the memory $\mathrm{CD} 8+$ lymphocytes produced in $\mathrm{YHV}-68$ preinfected mice were specific for PVM epitopes, raising the possibility that similar to RhCMV, $\gamma \mathrm{HV}-68$ can alter and potentially broaden the antigen specificity of lymphocyte responses upon a secondary viral challenge. It is interesting to speculate that because herpesviruses create lifelong infections that require ongoing T-cell activation (especially CD8+ T-cell activation) to maintain latency, that these cross-reactive and protective T-cell responses are likely actively boosted throughout life. The mechanisms for why herpesvirus infections yield broadly neutralizing CD8+ T-cell responses are unclear, but given the ability of herpesviruses to infect numerous cell types and their ability to generate multiple pathogen associated molecular patterns, they may be able to activate an especially wide variety of antigen presenting cells in many different tissue niches leading to broad responses.

The need for new strategies to produce a vaccine against RSV is pressing. RSV is one of the largest causes of hospitalization in newborn and young children with lower respiratory tract infections. Although a self-limiting infection, RSV has evolved several strategies that make it particularly good at evading the immune system. RSV replicates in superficial epithelial cells of the respiratory tract which ultimately leads to a less exuberant protective immune response. ${ }^{9}$ Thus, the ability to specifically bolster mucosal immune responses, e.g., memory or tissue resident lymphocytes, is paramount to increasing vaccine efficacy. Further, RSV infections are acquired early in life when the immature immune system is less able to mount robust antiviral responses. Unfortunately, this means that newborns and infants, who would be the target of RSV vaccination strategies, are unlikely to have acquired immune-shaping secondary herpesviral infections, which may partly explain the increased pathogenicity of RSV in these younger age groups. However, using a strategy to trick the immune system into mounting a herpesvirus-like immune response either preceding or concurrent with RSV vaccination by using a herpesvirus vector, or through careful selection of adjuvant, may prove efficacious. Another hurdle to RSV vaccine development is the observation that some children immunized with formalin inactivated RSV vaccines have gone on to develop severe pathology upon secondary viral challenge. ${ }^{1}$ Importantly, the study performed by Dourcy et al. demonstrated, using a formalin inactivated PVM vaccination model, that the altered immune responses fostered by $\mathrm{\gamma HV}-68$ preinfection alleviated this pathology reducing both damaging neutrophilic and eosinophilic infiltration while bolstering cytotoxic T-cell mediated protection.

\section{CONCLUDING REMARKS}

It is increasing becoming clear that our understanding of the immune system has been somewhat limited using single infection models and that the subtle shaping of immunity that occurs through successive rounds of viral and bacterial infections is often underappreciated. The article covered in this commentary gives insight into one such interaction, i.e., herpesvirus and RSV, which has important ramifications for both basic immunology and vaccine development.

\section{FUNDING}

This study was supported by NIH grant R35HL144481 (B.B.M.) and a Parker B. Francis fellowship (S.J.G.)

\section{ADDITIONAL INFORMATION}

Competing interests: The authors declare no competing interests.

Publisher's note Springer Nature remains neutral with regard to jurisdictional claims in published maps and institutional affiliations.

\section{REFERENCES}

1. Graham, B. S. Vaccine development for respiratory syncytial virus. Curr. Opin. Virol. 23, 107-112 (2017)

2. Ptaschinski, C. \& Lukacs, N. W. Early life respiratory syncytial virus infection and asthmatic responses. Immunol. Allergy Clin. North Am. 39, 309-319 (2019).

3. Schmidt, M. E. \& Varga, S. M. Cytokines and CD8 T cell immunity during respiratory syncytial virus infection. Cytokine (2018). https://doi.org/10.1016/j.cyto.2018.07.012.

4. Machiels, B. et al. A gammaherpesvirus provides protection against allergic asthma by inducing the replacement of resident alveolar macrophages with regulatory monocytes. Nat. Immunol. 18, 1310-1320 (2017).

5. Barton, E. S. et al. Herpesvirus latency confers symbiotic protection from bacterial infection. Nature 447, 326-329 (2007).

6. Barton, E. S., Rajkarnikar, S., Langston, P. K., Price, M. J. \& Grayson, J. M. Gammaherpesvirus latency differentially impacts the generation of primary versus secondary memory $\mathrm{CD} 8+\mathrm{T}$ cells during subsequent infection. J. Virol. 88, 12740-12751 (2014).

7. Picker, L. J., Hansen, S. G. \& Lifson, J. D. New paradigms for HIV/AIDS vaccine development. Annu Rev. Med 63, 95-111 (2012).

8. Hansen, S. G. et al. Cytomegalovirus vectors violate CD8+ T cell epitope recognition paradigms. Science 340, 1237874 (2013).

9. Fruh, K. \& Picker, L. CD8+ T cell programming by cytomegalovirus vectors: applications in prophylactic and therapeutic vaccination. Curr. Opin. Immunol. 47, 52-56 (2017). 